\title{
A rare case of primary pulmonary inflammatory pseudotumor-like follicular dendritic cell sarcoma successfully treated by lobectomy
}

\author{
Huayu He ${ }^{1}$, Qi Xue ${ }^{1}$, Fengwei Tan ${ }^{1}$, Lin Yang ${ }^{2}$, Xin Wang ${ }^{2}$, Yushun Gao ${ }^{1}$, Yousheng Mao ${ }^{1}$ Juwei Mu ${ }^{1}$, \\ Dali Wang ${ }^{1}$, Jun Zhao ${ }^{1}$, Liang Zhao ${ }^{1}$, Shugeng Gao ${ }^{1}$ \\ ${ }^{1}$ Department of Thoracic Surgery, National Cancer Center/National Clinical Research Center for Cancer/Cancer Hospital, Chinese Academy \\ of Medical Sciences and Peking Union Medical College, Beijing, China; ${ }^{2}$ Department of Pathology, National Cancer Center/National Clinical \\ Research Center for Cancer/Cancer Hospital, Chinese Academy of Medical Sciences and Peking Union Medical College, Beijing, China \\ Correspondence to: Shugeng Gao. Department of Thoracic Surgery, National Cancer Center/National Clinical Research Center for Cancer/Cancer \\ Hospital, Chinese Academy of Medical Sciences and Peking Union Medical College, Beijing, China. Email: gaoshugeng@vip.sina.com.
}

\begin{abstract}
Primary pulmonary inflammatory pseudotumor-like follicular dendritic cell sarcoma (IPT-like FDCS) is extremely rare. Here, we report a case of a 64-year-old female with primary pulmonary IPT-like FDCS. The patient was found to have a solid nodule in the right lower lobe (RLL) of the lung incidentally without any symptoms or signs of discomfort. The chest computed tomography (CT) showed that there was an irregular nodule in the basal segment of the RLL, approximately $2.0 \mathrm{~cm} \times 1.1 \mathrm{~cm} \times 1.0 \mathrm{~cm}$ in size, of $15 \mathrm{HU}$ in CT value. While the result of the fiberoptic bronchoscope-guided biopsy of the mass showed that there was inflammatory cell infiltration, no evidence of malignancy was found. After a thorough discussion of the multidisciplinary team, lobectomy of the RLL and systematic lymph node dissection were performed for the patient. Histologic analysis of the resected mass revealed infiltration of a large number of lymphocytes and plasma cells with the expression of CD21, CD23, CD35 were positive. In addition, the Epstein-Barr virus (EBV) probe in situ hybridization were positive. As a result, the diagnosis of EBV-positive IPT-like FDCS was strongly supported. No recurrence or any signs of metastasis were found during a 10-month follow-up time. As we have reported in this rare case, the diagnosis of primary pulmonary IPT-like FDCS should be considered even when there is only lymphoplasmacytic infiltration and no evidence of malignant tumor cells in the lung.
\end{abstract}

Keywords: Inflammatory pseudotumor-like follicular dendritic cell sarcoma (IPT-like-FDCS); primary; pulmonary; lobectomy

Submitted Jun 26, 2020. Accepted for publication Oct 26, 2020.

doi: 10.21037/atm-20-4965

View this article at: http://dx.doi.org/10.21037/atm-20-4965

\section{Introduction}

Inflammatory pseudotumor-like follicular dendritic cell sarcoma (IPT-like-FDCS) is a subset of follicular dendritic cell sarcoma (FDCS) originating from dendritic cells that is very rare and of low malignancy (1). Since it was identified as a unique entity in 2001, only approximately 42 cases of IPT-like FDCS have been reported in the English language literature $(2,3)$. The tumors mainly involve the liver and spleen and have not been reported in the lung. Herein, we report a rare case of primary pulmonary IPT- like FDCS successfully treated by lobectomy. Although the initial biopsy retrieved during bronchoscopy did not show malignant evidence, the final pathology confirmed IPT-like FDCS, which was associated with Epstein-Barr virus (EBV) positivity. We present the following case in accordance with the CARE reporting checklist (available at http://dx.doi. org/10.21037/atm-20-4965).

\section{Case presentation}

A 64-year-old woman approached our hospital to treat 

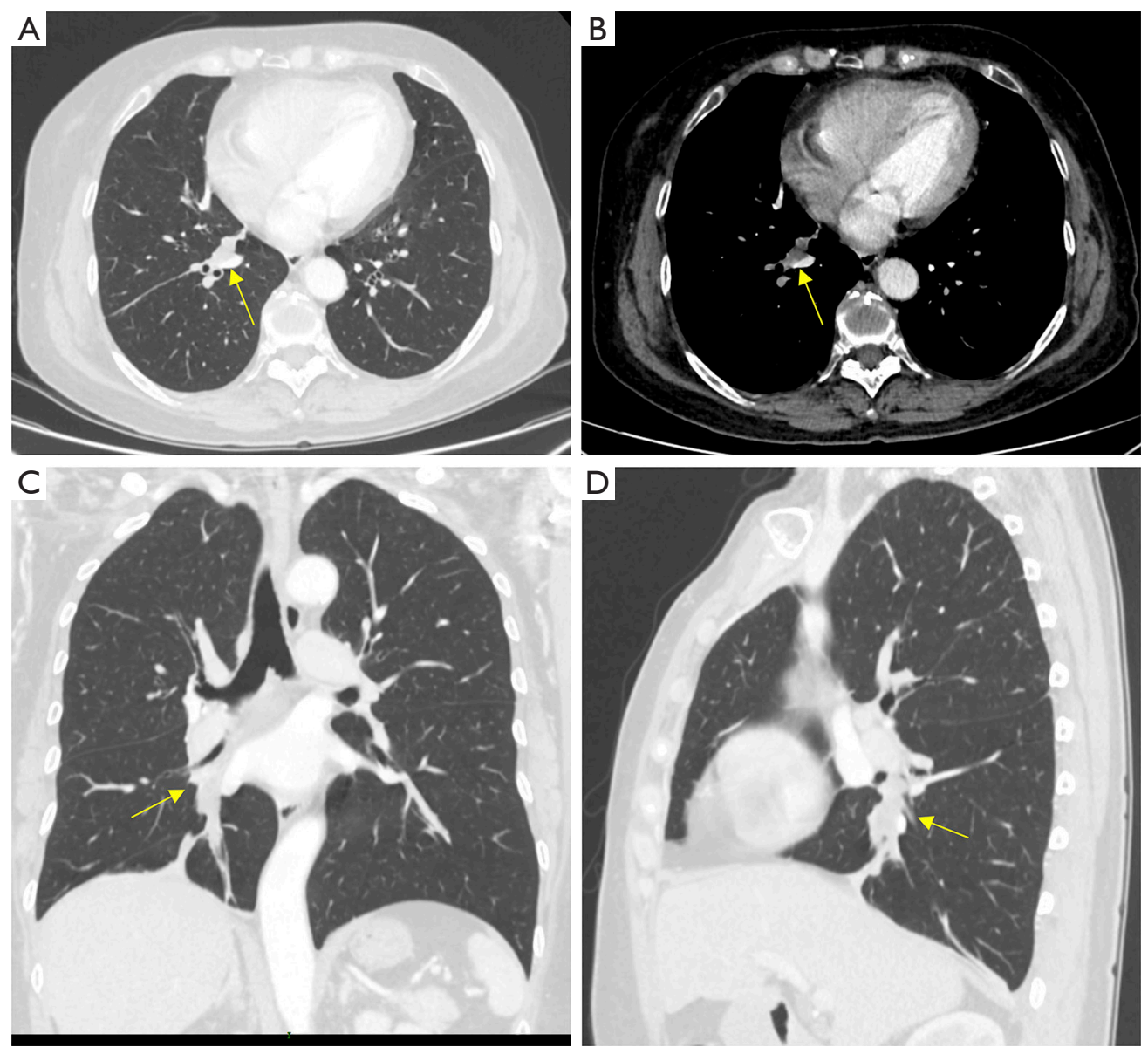

Figure 1 Chest enhanced computed tomography (CT) examination. The images show an irregular nodule in the basal segment of the RLL that is approximately $2.0 \mathrm{~cm} \times 1.1 \mathrm{~cm} \times 1.0 \mathrm{~cm}$ in size, with a low density and a CT value of approximately $15 \mathrm{HU}$. The lesions were not enhanced after enhancement. (A) Pulmonary window; (B) mediastinal window; (C) coronal image; (D) sagittal image. The arrows indicate the lesions.

a solid nodule in her right lower lobe (RLL) of the lung which was found incidentally during a normal physical examination a month ago. The chest computed tomography (CT) and physical examination showed irregular lowdensity nodule in the basal segment of the RLL of the lung, approximately $1.8 \mathrm{~cm} \times 1.0 \mathrm{~cm} \times 1.0 \mathrm{~cm}$ in size and of $13 \mathrm{HU}$ in CT value. She did not complain of any symptoms or discomfort, such as cough, expectoration, fever, exertion, chest distress, shortness of breath or weight loss. The patient received oral cefuroxime axetil tablet $(250.0 \mathrm{mg}$, twice daily) for 2 weeks under the guidance of the doctor, after which she came to our center and underwent chest CT again on August 16, 2019. The results showed that the irregular nodule in the basal segment of RLL was approximately $2.0 \mathrm{~cm} \times 1.1 \mathrm{~cm} \times 1.0 \mathrm{~cm}$ in size, with a low density and a CT value of approximately $15 \mathrm{HU}$. The right interlobular pleura were thickened. Low-density shadows could be seen in the bronchus of the RLL. No nodules or other lesions were found in the rest of the lung, and no lymphadenopathy was found in the mediastinum or hilum (Figure 1).

No significant abnormality was found on abdominal and pelvic CT, brain magnetic resonance imaging (MRI), radionuclide bone imaging, electrocardiogram (ECG) or pulmonary function examination. Almost all indexes of routine laboratory tests were in the normal range. The erythrocyte sedimentation rate and C-reactive protein level were also in the normal range. CA125, CYFRA21-1, NSE, SCC, CEA and ProGRP were all in the normal range in the tumor marker panel. Bronchoscopy showed 

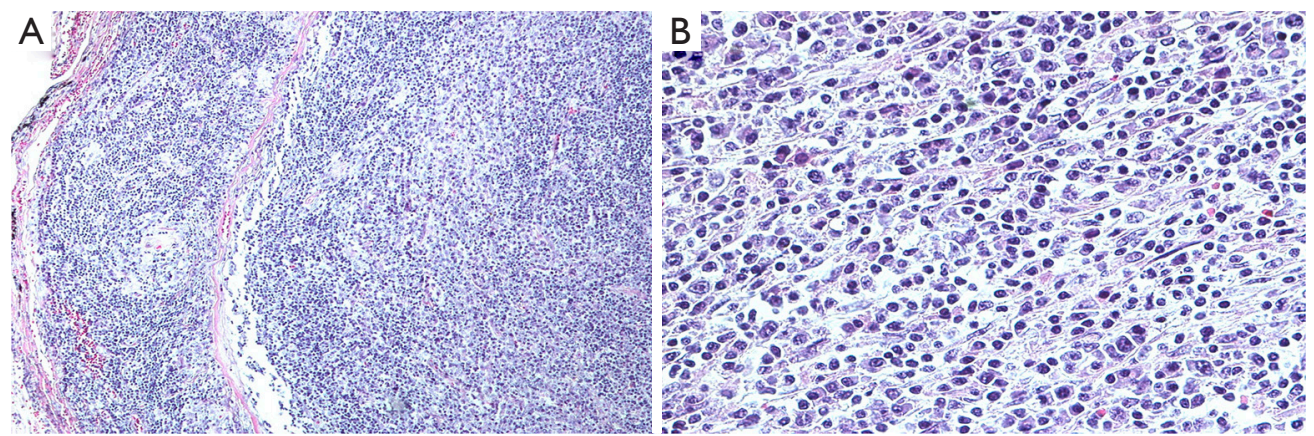

Figure 2 Composite H\&E staining: a large number of lymphoplasmacytic cells, accompanied by the proliferation of spindle cells. Original magnification: (A) $\times 100$; (B) $\times 400$.

necrosis on the surface of the basal segment of the RLL. No obvious abnormality was found in other parts of either lung. A fiberoptic bronchoscope-guided biopsy of the mass was performed before surgery. Under the bronchoscope, a suspicious new lesion was seen at the opening of the basal part of the RLL, and its surface was covered with necrotic material. The biopsy results showed that there was nonstructural necrotic material accompanied by inflammatory cell infiltration and small pieces of proliferative fibrous tissue, but there was no evidence of malignancy.

Despite the presence of the lesion on imaging, the biopsy results did not show evidence of malignancy. The patient refused bronchoscopy to re-examine the lesion and strongly desired surgical resection and a clear diagnosis, which made it difficult for the surgeons to decide her course of care. After several discussions by a multidisciplinary team, in view of the clear existence of a nodule in the lung of the patient, the possibility of a false negative bronchoscopy biopsy result, and the severe psychological pressure the disease was placing on the wellbeing of the patient, the decision was made to resect and determine the nature of the nodule.

During the operation, the nodule was found in the basal segment of the RLL, and the pleura of the visceral layer adjacent to the nodule was decreased. No other tissue was invaded. Because of the deep position of the nodule, it was impossible to perform pathological resection and biopsy. Lobectomy of the RLL and systematic lymph node dissection were performed directly. Fast-frozen pathological examination of the tumor and the edge of the bronchus was performed during the operation, but no malignant tumor cells were found.

Grossly, there was a polypoid mass in the bronchus of $2.0 \mathrm{~cm} \times 0.8 \mathrm{~cm} \times 0.8 \mathrm{~cm}$ in size; the cut side was gray and hard, the boundary with the surrounding bronchus mucosa was clear, and the lower lobe basal segment of the bronchus was blocked. The local focus involved the lung parenchyma and did not invade the visceral pleura. Microscopically, the lesion was characterized by a mixture of inflammatory cells and spindle cells. The spindle cells were scattered or arranged in disordered bundles. The cytoplasm of spindle cells was pale or eosinophilic. The boundaries of the cells were unclear. The nuclei were larger and longer than normal. Pleomorphic infiltration of inflammatory cells, mainly lymphocytes and plasma cells, was seen, with a small number of eosinophils and neutrophils mixed in with these cells (Figure 2). A total of 23 intact lymph nodes, including 2R, 4R, 7 and 9-14 areas, were found without tumor invasion, and some of them showed reactive hyperplasia. Immunohistochemical staining showed strongly positive expression of CD21 and CD23 and positive expression of CD35 and CD68 in tumor cells, which strongly supported the diagnosis of IPT-likeFDCS. Smooth muscle actin (SMA) and Desmin staining were strongly positive in the spindle cells but not in the areas where FDC markers such as CD21 were strongly expressed. Positive Kappa and Lambda expression and strongly positive CD38 expression were seen on plasma cells. The positive expression of CD68 and CD163 highlighted a histiocyte background. CD15, CD30, CD1a, S-100, ALK and AE1/ AE3 were negative. In addition, Ki-67 was positive, and its level was 30\% (Figure 3). Chromogenic in situ hybridization (CISH) for EBV-encoded RNA (EBER) was positive in all tumor cells (Figure 3). Therefore, there was enough evidence to diagnose the tumor as EBV-associated IPT-like-FDCS.

After the operation, the case was submitted to a multidisciplinary team for discussion. It was agreed that the tumor was small in size, with no obvious signs of metastasis; the lobectomy was adequate; and no additional 

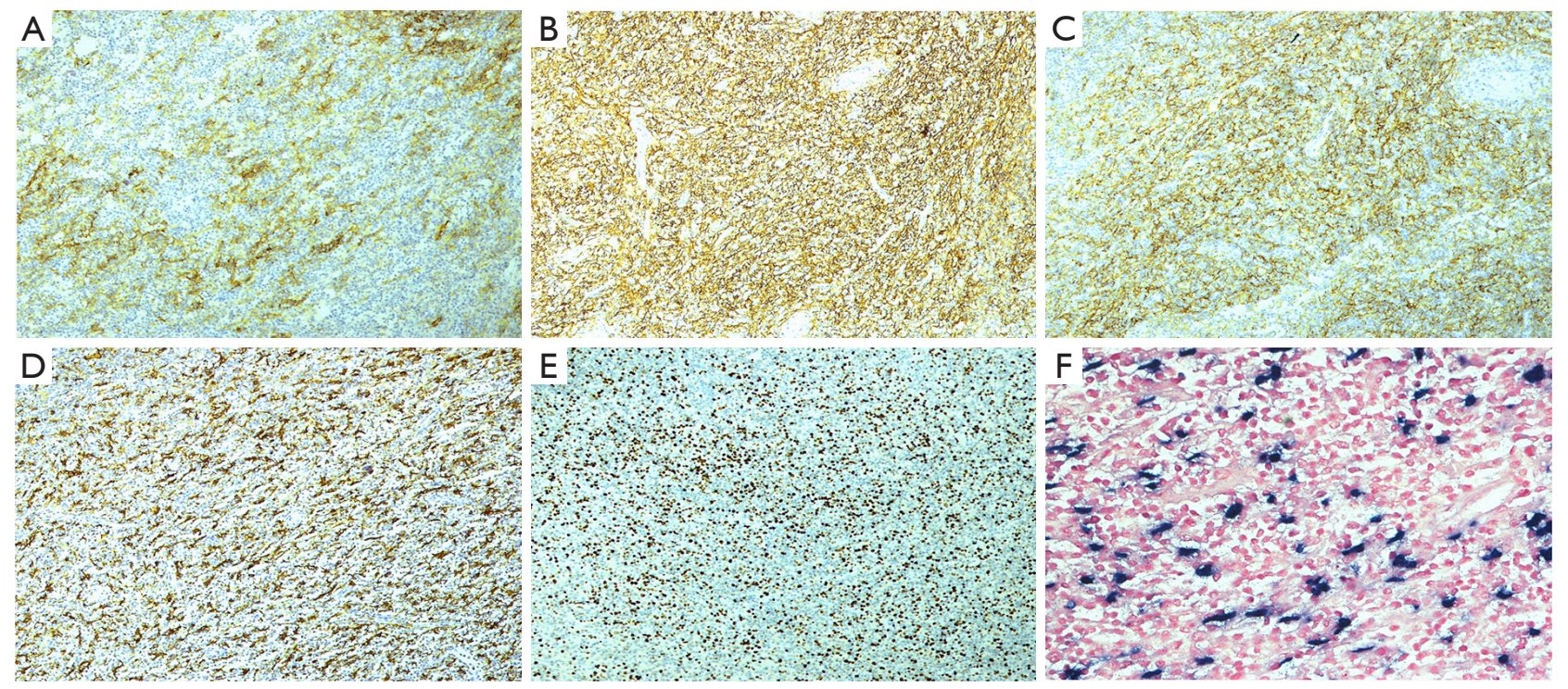

Figure 3 Follicular dendritic cell (FDC) marker. Immunohistochemistry staining, the Benchmark XT stainer. (A) CD21, strongly positive ( $\times 100)$; (B) CD23, strongly positive ( $\times 100)$; (C) CD35, strongly positive $(\times 100)$; (D) CD163, strongly positive $(\times 100)$; (E) Ki-67, 30\% ( $\times 100)$; (F) the EBV-coded RNA (EBER) was strongly positive by Chromogenic in situ hybridization (CISH) $(\times 400)$.

adjuvant treatment was needed at present. The patient was recommended to be monitored every 3 months for 2 years and every half a year after that, up to 5 years. No recurrence or other signs of metastasis were found in the chest CT examination 3 and 6 months after the operation.

All procedures performed in studies involving human participants were in accordance with the ethical standards of the institutional and/or national research committee(s) and with the Helsinki Declaration (as revised in 2013). Written informed consent was obtained from the patient.

\section{Discussion}

As far as we know from the English language literature, the primary pulmonary IPT-like FDCS reported in this case is the first of its kind. Of course, in the more than 30 previously reported cases of pulmonary FDCS (4), there may be cases of IPT-like FDCS that have not been fully diagnosed. The origin of IPT-like FDCS remains unclear. Most patients with IPT-like FDCS have been reported to have a positive EBER test. The primary pulmonary IPTlike-FDCS case in this study was also confirmed to be $\mathrm{EBV}$ positive. EBV infection occurs before the monoclonal proliferation of neoplastic follicular dendritic cells (FDCs) in hepatic IPT-like-FDCS (5). In addition, Choe et al. reported that a large number of immunoglobulin G4
(IgG4)-positive plasma cells were found in EBV-positive splenic IPT-like-FDCS, suggesting that EBV plays an important role in IPT-like-FDCS (6). However, the specific mechanism is still unclear, and further research is needed.

The histologic and immunohistochemical features of the case we reported were very typical, which also enabled the correct diagnosis via the postoperative histopathology results. IPT-like FDCS is histologically characterized by infiltration of a large number of lymphocytes and plasma cells; scattered spindle cells can be seen, the degree of nuclear atypia is variable, and some nuclei can appear abnormal or similar to Reed-Sternberg cells $(2,7)$. After immunohistochemical staining, CD21, CD23, CD35 and the EBV probe in situ hybridization were positive, which also strongly support the diagnosis (8).

Compared with FDCS, the inflammatory component of IPT-like-FDCS is more prominent in histology. Due to the more prominent inflammatory background and the lack of atypical cells, these lesions are often ignored or mistakenly considered inflammatory or reactive processes, such as inflammatory pseudotumors. In our case, the biopsy histopathology results were initially regarded as inflammatory disease because only a large number of infiltrating inflammatory cells and proliferative fibrous tissue were seen, which almost delayed the correct treatment. Before immunohistochemical staining, cases 
of suspected inflammatory myofibroblastic tumor (IMT), FDCS and large cell lymphoma are analyzed according to the histological morphology of the tumor. Specifically, the distribution of IPT-like-FDCS tumor cells is relatively loose, its background is rich in lymphocytes and plasma cells, and fusiform tumor cells have a certain degree of atypia, which is different from the findings in IMT (9). In addition, IPT-like-FDCS can express at least one characteristic FDC marker and show significantly EBVpositive expression in situ hybridization (2). In contrast, SMA is significantly expressed in IMT, which is negative for FDC markers and does not have EBV-positive characteristics $(9,10)$. The morphology of IPT-like FDCS is similar to that of diffuse large B cell lymphoma or anaplastic large cell lymphoma. However, the positive expression of CD68 and CD163 and the negative expression of specific B cell or $\mathrm{T}$ cell markers can help the differential diagnosis (11).

IPT-like FDCS is a low-grade malignant tumor with a good prognosis. Compared with traditional FDCS, IPT-like FDCS is more inert, with few instances of recurrence and metastasis (12). Surgery is an effective treatment method, and whether adjuvant radiotherapy and chemotherapy should be added after surgery remains controversial. Saygin et al.'s study of 343 cases of FDCS found that there was no significant difference in the survival effect of adjuvant radiotherapy (4). Surgical resection is the primary choice for the treatment of pulmonary FDCS (13). However, Shah et al. reported a case of FDCS in the lung. The patient had just begun to receive adriamycin-based chemotherapy and then experienced pneumonectomy due to lack of response. Lymph node metastasis was found by pathology, and distant metastasis occurred 19 months later (14). Almost all of the cases of inflammatory pseudotumors, such as FDCS, reported in the literature have occurred in the liver and spleen. Complete resection and local lymph node dissection can effectively reduce tumor recurrence and metastasis (8). In our case, lobectomy was performed without lymph node metastasis in the lung, and no adjuvant treatment was recommended after the operation. There was no evidence of recurrence and metastasis after 10 months of follow-up, and we will continue to follow up with this patient regularly in the future.

In conclusion, as we have reported in this rare case, the diagnosis of primary pulmonary IPT-like-FDCS should be considered even when there is only lymphoplasmacytic infiltration and no evidence of malignant tumor cells in the lung.

\section{Acknowledgments}

Funding: This study was supported by the Institutional Fundamental Research funds (2018PT32033), the Ministry of Education innovation Team development project (IRT17R10).

\section{Footnote}

Reporting Checklist: The authors have completed the CARE reporting checklist. Available at http://dx.doi.org/10.21037/ atm-20-4965

Conflicts of Interest: All authors have completed the ICMJE uniform disclosure form (available at http://dx.doi. org/10.21037/atm-20-4965). The authors have no conflicts of interest to declare.

Ethical Statement: The authors are accountable for all aspects of the work in ensuring that questions related to the accuracy or integrity of any part of the work are appropriately investigated and resolved. All procedures performed in studies involving human participants were in accordance with the ethical standards of the institutional and/or national research committee(s) and with the Helsinki Declaration (as revised in 2013). Written informed consent was obtained from the patient.

Open Access Statement: This is an Open Access article distributed in accordance with the Creative Commons Attribution-NonCommercial-NoDerivs 4.0 International License (CC BY-NC-ND 4.0), which permits the noncommercial replication and distribution of the article with the strict proviso that no changes or edits are made and the original work is properly cited (including links to both the formal publication through the relevant DOI and the license). See: https://creativecommons.org/licenses/by-nc-nd/4.0/.

\section{References}

1. Li L, Shi YH, Guo ZJ, et al. Clinicopathological features and prognosis assessment of extranodal follicular dendritic cell sarcoma. World J Gastroenterol 2010;16:2504-19.

2. Cheuk W, Chan JK, Shek TW, et al. Inflammatory pseudotumor-like follicular dendritic cell tumor: a distinctive low-grade malignant intra-abdominal neoplasm 
with consistent Epstein-Barr virus association. Am J Surg Pathol 2001;25:721-31.

3. Mograbi M, Stump MS, Luyimbazi DT, et al. Pancreatic inflammatory pseudotumor-like follicular dendritic cell tumor. Case Rep Pathol 2019;2019:2648123.

4. Saygin C, Uzunaslan D, Ozguroglu M, et al. Dendritic cell sarcoma: a pooled analysis including 462 cases with presentation of our case series. Crit Rev Oncol Hematol 2013;88:253-71.

5. Shia J, Chen W, Tang LH, et al. Extranodal follicular dendritic cell sarcoma: clinical, pathologic, and histogenetic characteristics of an underrecognized disease entity. Virchows Arch 2006;449:148-58.

6. Choe JY, Go H, Jeon YK, et al. Inflammatory pseudotumor-like follicular dendritic cell sarcoma of the spleen: a report of six cases with increased IgG4-positive plasma cells. Pathol Int 2013;63:245-51.

7. Tregnago AC, Morbeck DL, D'Almeida Costa F, et al. Inflammatory pseudotumor-like follicular dendritic cell tumor: an underdiagnosed neoplasia. Applied Cancer Research 2017;37:45.

8. Zhang BX, Chen ZH, Liu Y, et al. Inflammatory

Cite this article as: He H, Xue Q, Tan F, Yang L, Wang X, Gao Y, Mao Y, Mu J, Wang D, Zhao J, Zhao L, Gao S. A rare case of primary pulmonary inflammatory pseudotumorlike follicular dendritic cell sarcoma successfully treated by lobectomy. Ann Transl Med 2021;9(1):77. doi: 10.21037/atm-204965 pseudotumor-like follicular dendritic cell sarcoma: A brief report of two cases. World J Gastrointest Oncol. 2019;11:1231-9.

9. Neuhauser TS, Derringer GA, Thompson LD, et al. Splenic inflammatory myofibroblastic tumor (inflammatory pseudotumor): a clinicopathologic and immunophenotypic study of 12 cases. Arch Pathol Lab Med 2001;125:379-85.

10. Gleason BC, Hornick JL. Inflammatory myofibroblastic tumours: where are we now? J Clin Pathol 2008;61:428-37.

11. Denning KL, Olson PR, Maley RH Jr, et al. Primary pulmonary follicular dendritic cell neoplasm: a case report and review of the literature. Arch Pathol Lab Med 2009;133:643-7.

12. Ge R, Liu C, Yin X, et al. Clinicopathologic characteristics of inflammatory pseudotumor-like follicular dendritic cell sarcoma. Int J Clin Exp Pathol 2014;7:2421-9.

13. Kovács RB, Sattar HA, Krausz T, et al. Primary follicular dendritic cell sarcoma of the lung. Histopathology 2006;49:431-3.

14. Shah RN, Ozden O, Yeldandi A, et al. Follicular dendritic cell tumor presenting in the lung: a case report. Hum Pathol 2001;32:745-9. 